\title{
Efeito do sistema de preparo do solo sobre características agronômicas da mandioca (Manihot esculenta Crantz)
}

\author{
Manoel G. Pequeno ${ }^{1}$, Pedro S. Vidigal Filho ${ }^{1}$, Cássio Tormena ${ }^{1}$, Marcus V. Kvitschal ${ }^{2} \&$ Marcelo Manzotti ${ }^{3}$
}

\begin{abstract}
RESUMO
Neste trabalho se propôs avaliar o efeito de sistemas de preparo de um Latossolo Vermelho distrófico sobre as características agronômicas da mandioca durante quatro anos agrícolas de 1999/2000, 2000/2001, 2001/2002 e 2002/2003, em Araruna, Noroeste do Estado do Paraná. O delineamento experimental foi o de blocos completos casualizados com oito repetições. Os tratamentos se constituíram de três sistemas de preparo do solo: plantio direto; preparo mínimo (escarificador com rolo nivelador) e preparo convencional (arado de aivecas + grade niveladora). O sistema de preparo convencional do solo propiciou maior altura de plantas e maior produção de parte aérea e de raízes tuberosas em relação ao preparo mínimo e ao plantio direto. O teor de massa seca nas raízes tuberosas não foi influenciado pelos sistemas de preparo de solo.
\end{abstract}

Palavras-chave: mandioca, preparo convencional, preparo mínimo, plantio direto

\section{Effect of soil tillage on agronomic characteristics of cassava (Manihot esculenta Crantz)}

\begin{abstract}
The purpose of this research was to study the effect of soil tillage systems in a distrophic Red Latosol on agronomic characteristics of cassava during four growth cycles (1999/2000, 2000/2001, 2001/2002 and 2002/2003), in Araruna, northwest region of Paraná state, Brazil. The experimental design was in completely randomized blocks with eight replications. The treatments consisted of three soil tillage systems: no-tillage, minimum tillage using chiseling and conventional tillage (moldboard plow and disking). The conventional tillage system resulted in taller plants and higher storage roots yield in relation to the minimum and no-tillage system. The root dry matter content was not influenced by the tillage systems used.
\end{abstract}

Key words: cassava, conventional tillage, minimum tillage, no-tillage 


\section{INTRODUÇÃO}

A espécie Manihot esculenta Crantz é encontrada entre os paralelos $30^{\circ}$ de latitude Norte e Sul, com maior concentração entre os paralelos $20^{\circ} \mathrm{N}$ e $20^{\circ} \mathrm{S}$, cultivada em altitudes de até 2.300 m, próximo ao Equador (Cock, 1982). De acordo com FAO (2006), a produção mundial de mandioca vem crescendo, em especial nos países africanos, onde a cultura se constitui na principal fonte energética de um grande contingente populacional. O Brasil, segundo maior produtor mundial, produziu no agrícola de 2005/06 mais de 27,6 milhões de toneladas de raízes tuberosas de mandioca em aproximadamente 1,9 milhões de hectares (FAO, 2006).

O Estado do Paraná, por sua vez, tem se destacado por se tratar de um dos principais produtores de mandioca do país, tendo apresentado, em 2005/06, uma produção de 4,2 milhões de toneladas, proveniente de uma área cultivada de 205 mil ha (Groxko, 2006).

Nas regiões Noroeste e Centro Oeste do Estado do Paraná, constituídas, em sua maioria, de solos de textura média a arenosa, com reduzida capacidade de retenção de água e elevada suscetibilidade a erosão (Fidalski, 1997), se observa uma concentração maior da cultura da mandioca (Groxko, 2006). Nessas regiões, o cultivo intensivo tem propiciado redução gradativa da produtividade da cultura da mandioca que tem, por característica, apresentar crescimento inicial lento, o que resulta em elevada perda de solo decorrente do processo erosivo (Souza, 1986).

Há indicações de que, para algumas culturas, o plantio direto e o preparo mínimo propiciam produtividades mais elevadas em comparação com o preparo convencional do solo (Souza \& Carvalho, 1995); entretanto, são poucos os resultados de avaliação dos efeitos dos sistemas de preparo do solo sobre a produtividade da cultura da mandioca no Brasil (Souza, 1986; Carvalho et al., 1988; Souza \& Carvalho, 1995; Oliveira et al., 2001) e, em particular, na região Noroeste do Estado do Paraná, uma das principais produtoras desta cultura no Brasil.

Desta forma se objetivou, com este estudo, avaliar o comportamento de algumas características agronômicas da mandioca submetida a três sistemas de preparo de solo, ao longo de quatro cultivos sucessivos.

\section{MATERIAL E MÉTODOS}

Os experimentos foram instalados em Araruna, Noroeste do Estado do Paraná, nos anos agrícolas de 1999/2000, 2000/ 2001, 2001/2002 e 2002/2003. O município de Araruna se situa a $23^{\circ} 55^{\prime}$ de Latitude Sul e a $52^{\circ} 30^{\prime}$ de Longitude Oeste de Greenwich, com altitude média de 660 m; o clima é mesotérmico, sem estação seca, com verão quente, temperatura anual média de $21,5^{\circ} \mathrm{C}$, precipitação média anual de $1.617 \mathrm{~mm}$ e umidade relativa média anual de 62\% (Godoy et al., 1976). Os dados referentes às temperaturas máxima e mínima e à precipitação pluvial no período experimental, encontram-se na Figura 1.

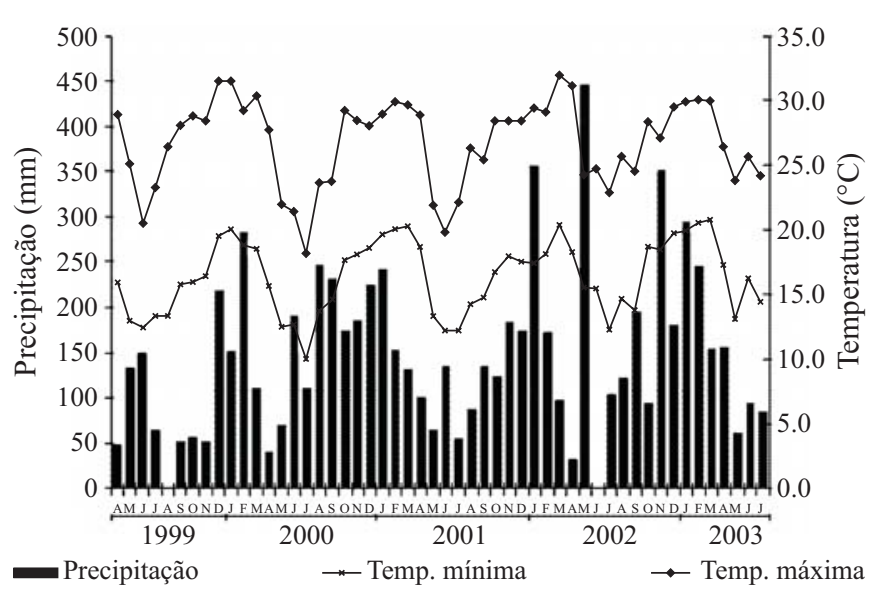

Figura 1. Valores totais mensais de precipitação pluvial e temperatura máxima e mínima observados no período de abril de 1999 a julho de 2003. Araruna, PR

O solo da área experimental, que vinha sendo explorado durante três anos com a cultura da soja em sistema de plantio direto, é classificado como Latossolo Vermelho distrófico, textura Franco Argilo-Arenosa (EMBRAPA, 1999). A análise química de amostras de material de solo coletadas na camada de 0,0-0,20 m por ocasião da implantação dos experimentos apresentou os seguintes resultados: $\mathrm{pH}$ $\left(\mathrm{H}_{2} \mathrm{O}\right)=5,2 ; \mathrm{Al}^{+3}=0,0 \mathrm{cmol}_{\mathrm{C}} \mathrm{dm}^{-3} ; \mathrm{Ca}^{+2}=1,49 \mathrm{cmol}_{\mathrm{C}} \mathrm{dm}^{-3}$; $\mathrm{Ca}^{+2}+\mathrm{Mg}^{+2}=2,44 \mathrm{cmol}_{\mathrm{C}} \mathrm{dm}^{-3} ; \mathrm{K}^{+}=0,32 \mathrm{cmol}_{\mathrm{C}} \mathrm{dm}^{-3}$; $\mathrm{P}=5,0 \mathrm{mg} \mathrm{dm}^{-3} ; \mathrm{Fe}=44,81 \mathrm{mg} \mathrm{dm}^{-3}, \mathrm{Cu}=1,2 \mathrm{mg} \mathrm{dm}^{-3}$; $\mathrm{Zn}=0,9 \mathrm{mg} \mathrm{dm}^{-3}$ e $\mathrm{C}=13,54 \mathrm{~g} \mathrm{dm}^{-3}$.

A implantação dos experimentos se iniciou com a correção do solo para $60 \%$ de saturação por bases, conforme Lorenzi \& Dias (1993), utilizando-se calcário dolomítico. Em abril de 1999, julho de 2000 e agosto de 2001 e de 2002, efetuou-se o plantio da cultura de inverno; nos dois primeiros períodos de avaliação procedeu-se à semeadura direta de aveia preta (Avena strigosa, Schreb) associada ao nabo forrageiro (Raphanus sativus L. var. oleiferus Metzg), enquanto nos anos seguintes se utilizou o milheto (Pennisetum americanum L.). A adubação de plantio da cultura de inverno (aveia/nabo e milheto) foi efetuada com o correspondente a $96 \mathrm{~kg} \mathrm{ha}^{-1}$ de $\mathrm{P}_{2} \mathrm{O}_{5}, 30 \mathrm{~kg} \mathrm{ha}^{-1}$ de $\mathrm{K}_{2} \mathrm{O}$ e $25 \mathrm{~kg} \mathrm{ha}^{-1}$ de $\mathrm{N}$, e adubação de cobertura com $25 \mathrm{~kg} \mathrm{ha}^{-1}$ de $\mathrm{N}$. Por ocasião da floração plena da aveia/nabo ou do milheto, os mesmos passaram por um processo de dessecação utilizando-se de 1,5 L ha-1 Gramoxone 200.

Os tratamentos consistiram de três sistemas de preparo do solo: a) preparo convencional (PC): aração com arado de três aivecas, reversível, com largura de corte $1,20 \mathrm{~m}$ e profundidade de trabalho de 0,20 a $0,25 \mathrm{~m}$, seguido de uma gradagem com grade niveladora de arraste; b) preparo mínimo (PM): revolvimento do solo apenas com escarificador Ikeda, modelo DP-220 M, de arrasto, com três hastes de curvatura helicoidal, espaçamento entre hastes de $0,60 \mathrm{~m}$, ponteiras retas de $100 \mathrm{~mm}$ de espessura, sem asas, munido de rolo nivelador acoplado na parte posterior para o nivelamento do solo e quebra de torrões, e com largura de corte de 2,5 m; c) plantio direto (PD): nenhum preparo prévio do solo com plantio direto das manivas a profundidade média de $0,10 \mathrm{~m}$ 
por meio de plantadora de mandioca semi-mecanizada, marca Plant Center, Modelo PC 20, com capacidade de plantio simultâneo de duas linhas.

O delineamento experimental utilizado foi o de blocos completos casualizados, com oito repetições, perfazendo o total de 24 unidades experimentais. Com o objetivo de facilitar a aplicação e distribuição dos tratamentos, prepararamse áreas de 8,0 m de largura x 150 m de comprimento, atingindo o total de $1.200 \mathrm{~m}^{2}$ para cada tratamento, conforme metodologia utilizada por Souza et al. (1994), Souza \& Carvalho (1995) e por Oliveira et al. (2001). Dentro de cada uma dessas áreas, correspondentes aos diferentes tratamentos, foram demarcadas as unidades experimentais (parcelas) após o plantio mecanizado da mandioca; as referidas parcelas apresentaram dimensões de 5 x 15 m (75 m²), com área útil de $30 \mathrm{~m}^{2}$; o espaçamento utilizado foi de $1,0 \mathrm{~m}$ entre linhas e de $0,80 \mathrm{~m}$ entre plantas na linha de plantio, com o objetivo de se estabelecer uma população de 12.500 plantas ha-1. As cultivares de mandioca utilizadas foram a Espeto (nos dois primeiros anos agrícolas) e a Fécula Branca (nos dois últimos), em função das suas características agronômicas (Vidigal Filho et al., 2000) e da disponibilidade de ramas de boa qualidade para o plantio.

Com ramas oriundas de lavouras com cerca de 9-10 meses de ciclo, colhidas no município de Araruna, efetuou-se o plantio da mandioca em 10/10/1999, 21/10/2000, 21/11/2001 e 11/12/2002; as manivas apresentaram tamanho médio de 0,15-0,20 m, conforme recomendações de Lorenzi \& Dias (1993).

Na condução da cultura, que não recebeu nenhuma adubação NPK tendo em vista o aproveitamento do efeito residual da adubação fornecida às culturas de inverno, foram utilizados os tratos culturais comuns à cultura mandioca. Por ocasião da colheita (8 a 9 meses após a emergência das plantas) dos experimentos, avaliaram-se as características altura de plantas (ALP), produção de parte aérea (PPA), produção de raízes tuberosas (PR) e o teor de matéria seca nas raízes tuberosas (MS). O teor de matéria seca foi determinado pelo método da balança hidrostática, conforme Grosmann \& Freitas (1950).

Os dados experimentais foram submetidos a análise de variância individual a fim de se verificar o efeito dos tratamentos (sistemas de preparo do solo), e a homogeneidade entre as variâncias residuais. Admitiram-se os anos de avaliação que mostraram razão entre a maior e a menor variância residual de até 7:1, conforme Cruz \& Regazzi (2001). As médias dos tratamentos foram comparadas pelo teste de Tukey a 5\% de probabilidade de erro. Os sistemas de preparo foram considerados como de efeito fixo e os anos de avaliação o foram como de efeito aleatório.

\section{RESULTADOS E DISCUSSÃO}

A análise de variância conjunta mostrou efeito significativo $(\mathrm{P} \leq 0,05)$ da interação entre os sistemas de preparo do solo e os anos agrícolas para todas as características avaliadas (Tabela 1) indicando a necessidade de desdobramento em
Tabela 1. Resumo da análise de variância conjunta para altura de plantas $(A L P)$, produção de parte aérea (PPA), produção de raízes tuberosas (PR) e teor de matéria seca (MS) nas raízes tuberosas da mandioca sob três sistemas de preparo de solo, nos anos agrícolas de 1999/00, 2000/01, 2001/02 e 2002/03. Araruna, PR

\begin{tabular}{lcccrr}
\hline \multirow{2}{*}{ F.V. } & \multirow{2}{*}{ GL } & \multicolumn{4}{c}{ Quadrado médio } \\
\cline { 3 - 6 } & & ALP (m) & PPA (t ha-1 & PR (t ha-1) & MS (\%) \\
Bloco/ano & 28 & 0,013 & 5,816 & 9,988 & 1,147 \\
Sistema de Preparo & 2 & 0,165 & 84,032 & 345,625 & 0,867 \\
Anos & 3 & 0,477 & 431,971 & $1.561,172$ & 65,631 \\
Sist. Prep. x Ano & 6 & $0,051^{*}$ & $26,085^{*}$ & $79,584^{*}$ & $1,599^{*}$ \\
Resíduo & 56 & 0,006 & 2,5172 & 5,303 & 0,556 \\
C.V. (\%) & & 5,08 & 16,63 & 10,90 & 2,14 \\
Média Geral & & 1,52 & 9,54 & 21,13 & 34,83 \\
\hline
\end{tabular}

* Significativo $(P \leq 0,05)$ pelo teste $F$

cada ano de avaliação. A variabilidade na disponibilidade de água, devido à variação da precipitação pluvial entre os anos agrícolas estudados (Figura 1), foi um dos fatores que determinaram a significância da interação.

Com base nos resultados referentes à comparação entre as médias de altura de plantas no primeiro e terceiro anos de avaliação (Tabela 2), pode-se verificar que as médias do PC foram superiores ao PM e ao PD; no ano 2, o PM proporcionou maior altura de plantas em relação aos demais sistemas de preparo; já no ano 4 e na média dos quatro anos de avaliação, o PC e o PM proporcionaram maior crescimento das plantas enquanto o PD não mostrou diferença significativa $(\mathrm{P}>0,05)$ apenas com o $\mathrm{PM}$; referidos resultados foram similares aos obtidos por Oliveira et al. (2001) e Souza et al. (1994).

A redução no crescimento das plantas de mandioca no PD pode estar associada à maior densidade na camada superficial do solo, o que propicia aumento na impedância mecânica relacionada ao crescimento inicial do sistema radicular, com conseqüente prejuízo no crescimento da parte aérea (Oliveira et al., 2001).

A avaliação das condições físicas do solo da área experimental realizada por Watanabe et al. (2002) e por Cavalieri (2004) nos dois primeiros anos de cultivo, indicou maiores valores de densidade do solo quando o mesmo foi submetido ao PD, seguido do PM e do PC; esses resultados explicam o menor crescimento das plantas nos sistemas que promoveram menor revolvimento do solo. A maior altura de plantas no PM no ano 2 pode ser decorrente da melhor distribuição de chuvas verificada neste período (Figura 1).

Por outro lado, e em situação de maior variabilidade hídrica associada ao clima, sistemas de preparo que resultem em menor impedância na camada superficial determinam o melhor desempenho da mandioca, em termos de altura de plantas, como verificado no PC. A severidade das limitações observadas no PD implica em reduzido crescimento das plantas, independentemente das condições climáticas.

Conforme Passioura \& Gardner (1990), a presença de camadas compactadas pode resultar em valores restritivos de resistência do solo, o que implica na redução do crescimento das plantas devido ao ambiente desfavorável ao 
Tabela 2. Médias de altura de plantas, produção de parte aérea, produção de raízes e teor de matéria seca nas raízes tuberosas da mandioca, sob três sistemas de preparo de solo, nos anos agrícolas de 1999/00, 2000/01, 2001/02 e 2002/03

\begin{tabular}{|c|c|c|c|c|c|c|c|c|c|c|}
\hline \multirow{2}{*}{ Sistema de Preparo } & \multicolumn{5}{|c|}{ Altura de plantas (m) } & \multicolumn{5}{|c|}{ Produção de parte aérea $\left(t \mathrm{ha}^{-1}\right)$} \\
\hline & Ano 1 & Ano 2 & Ano 3 & Ano 4 & Média & Ano 1 & Ano 2 & Ano 3 & Ano 4 & Média \\
\hline P. Convencional (PC) & $1,84 \mathrm{a}$ & $1,58 \mathrm{~b}$ & $1,56 \mathrm{a}$ & $1,40 \mathrm{a}$ & $1,60 \mathrm{a}$ & $17,56 \mathrm{a}$ & $14,30 \mathrm{a}$ & $8,67 \mathrm{a}$ & $5,06 \mathrm{a}$ & $11,40 \mathrm{a}$ \\
\hline P. Mínimo (PM) & $1,59 \mathrm{~b}$ & $1,70 \mathrm{a}$ & $1,44 \mathrm{~b}$ & $1,36 \mathrm{ab}$ & $1,52 a b$ & $10,55 \mathrm{~b}$ & $13,34 \mathrm{a}$ & $6,66 \mathrm{~b}$ & $4,69 \mathrm{a}$ & $8,81 \mathrm{~b}$ \\
\hline P. Direto (PD) & $1,56 \mathrm{~b}$ & $1,55 \mathrm{~b}$ & $1,41 \mathrm{~b}$ & $1,29 \mathrm{~b}$ & $1,45 \mathrm{~b}$ & $9,81 \mathrm{~b}$ & $12,78 \mathrm{a}$ & $7,06 \mathrm{ab}$ & $3,99 \mathrm{a}$ & $8,41 \mathrm{~b}$ \\
\hline $\mathrm{F}_{(\mathrm{QMT} / \mathrm{QMR})}$ & $33,22^{\star}$ & $8,31^{*}$ & $8,00^{*}$ & $3,98 *$ & - & $58,07^{*}$ & $1,89^{\text {ns }}$ & $3,58^{*}$ & $0,93^{\text {ns }}$ & - \\
\hline C.V. $(\%)$ & 4,66 & 4,80 & 5,26 & 5,73 & 5,08 & 12,55 & 11,78 & 21,24 & 34,64 & 16,63 \\
\hline Média Geral & 1,66 & 1,61 & 1,47 & 1,35 & 1,52 & 12,64 & 13,47 & 7,47 & 4,58 & 9,54 \\
\hline \multirow{2}{*}{ Sistema de Preparo } & \multicolumn{5}{|c|}{ Produção de raízes tuberosas $\left(t^{h a-1}\right)$} & \multicolumn{5}{|c|}{ Teor de matéria seca (\%) } \\
\hline & Ano 1 & Ano 2 & Ano 3 & Ano 4 & Média & Ano 1 & Ano 2 & Ano 3 & Ano 4 & Média \\
\hline P. Convencional (PC) & 35,97 a & $32,18 \mathrm{a}$ & 16,79 a & 13,83 a & 24,69 a & $36,90 \mathrm{a}$ & 34,89 a & 35,49 a & $32,11 \mathrm{a}$ & $34,85 \mathrm{a}$ \\
\hline P. Mínimo (PM) & $25,64 \mathrm{~b}$ & $27,13 \mathrm{~b}$ & $16,58 \mathrm{a}$ & $12,56 a b$ & $20,48 \mathrm{~b}$ & $36,69 a b$ & $34,58 \mathrm{a}$ & $35,70 \mathrm{a}$ & $32,95 \mathrm{a}$ & $34,98 \mathrm{a}$ \\
\hline P. Direto (PD) & $21,12 \mathrm{c}$ & $25,67 \mathrm{~b}$ & $15,76 \mathrm{a}$ & $10,31 \mathrm{~b}$ & 18,22 b & $35,91 \mathrm{~b}$ & $34,04 \mathrm{a}$ & $35,70 \mathrm{a}$ & $32,95 \mathrm{a}$ & $34,65 \mathrm{a}$ \\
\hline$F_{(Q M T / Q M R)}$ & $87,35^{\star}$ & $17,63^{\star}$ & $0,45^{\mathrm{ns}}$ & $4,79^{*}$ & - & $3,90^{*}$ & $2,64^{\mathrm{ns}}$ & $0,22^{\mathrm{ns}}$ & $3,43^{\text {ns }}$ & - \\
\hline C.V. $(\%)$ & 8,35 & 8,13 & 14,06 & 18,83 & 10,90 & 2,04 & 2,16 & 2,09 & 2,28 & 2,14 \\
\hline Média Geral & 27,58 & 28,33 & 16,38 & 12,23 & 21,13 & 36,50 & 34,50 & 35,63 & 32,67 & 34,83 \\
\hline
\end{tabular}

*Significativo $(P \leq 0,05)$ pelo teste $F$; nsNão significativo; Médias seguidas da mesma letra na coluna não diferem entre si $(P>0,05)$ pelo teste de Tukey

desenvolvimento de suas raízes. Quando em condições de excessiva resistência a penetração, as raízes das plantas enviam sinais metabólicos inibitórios para as folhas, através de compostos químicos, tais como o ácido abcísico, liberado pelas raízes na corrente transpiratória, resultando em menor crescimento das plantas (Ismail \& Davies, 1998).

Quanto à produção de parte aérea (Tabela 2), pode-se observar que no ano 1 o PC proporcionou superioridade de 39,9 e 44,1\% em relação aos sistemas de PM e PD, respectivamente; no ano 3, o PC não diferiu do $\mathrm{PD}(\mathrm{P}>0,05)$ mas se mostrou superior ao PM; por sua vez, nos anos 2 e 4 os tratamentos não diferiram entre si $(\mathrm{P}>0,05)$; no ano 2 , os tratamentos não diferiram entre si devido, provavelmente, às boas condições climáticas que propiciaram boa resposta de crescimento das plantas (Figura 1), independente do sistema de preparo; entretanto, no ano 4 não houve diferença entre os tratamentos em função, supostamente, do atraso no plantio da cultura. Na média dos quatro anos, o PC superou o $\mathrm{PM}$ e o PD, sendo que esses dois últimos não diferiram entre si $(\mathrm{P}>0,05)$. Estes resultados foram similares aos apresentados por Souza et al. (1994), que encontraram menor produção de parte aérea de plantas de mandioca ao utilizarem o plantio em covas (sistema de preparo que envolve mínimo revolvimento do solo). Por outro lado, os resultados obtidos diferem daqueles apresentados por Oliveira et al. (2001), que obtiveram maior produção de parte aérea de mandioca utilizando o sistema de preparo mínimo. De modo geral, o PC mostrou médias mais elevadas de altura de plantas e de produção de parte aérea (Tabela 2), seguido do PM e do PD. Visto que Watanabe et al. (2002) e Cavalieri (2004) verificaram maior densidade do solo nos sistemas de PD, PM e $\mathrm{PC}$, respectivamente, fica evidente que as plantas de mandioca, quando cultivadas sob um sistema de preparo que envolve maior revolvimento do solo, devem apresentar maior crescimento e desenvolvimento da parte aérea. Sob condições de maior densidade do solo nas camadas superficiais, existe a possibilidade das plantas terem sido submetidas a estresses físicos, o que pode comprometer a produtividade das culturas, caso o estresse ocorra em estágio fenológico crítico (Domzal \& Slowinska-Jurkiewicz, 1987).

Quanto à produção de raízes tuberosas (Tabela 2) no ano 1 os três sistemas de preparo mostraram diferença significativa entre si $(\mathrm{P} \leq 0,05)$, de forma que o PC propiciou maior média, seguido do PM e do PD, respectivamente; no ano 2 os sistemas de PM e de PD foram significativamente $(\mathrm{P} \leq 0,05)$ superados pelo $\mathrm{PC}$; no ano 3 os tratamentos não diferiram entre si $(\mathrm{P}>0,05)$, o que pode ser atribuído às severas restrições hídricas associadas à ocorrência de veranico em fevereiro/março e abril/maio de 2002 (Figura 1). Por sua vez, no ano 4 a produção no PC não diferiu estatisticamente $(\mathrm{P}>0,05)$ daquela obtida no PM mas superou a produção obtida no PD. Considerando-se a produção média nos quatro anos de avaliação, conclui-se que o PC foi superior em 20,55 e 35,5\% em relação ao PM e ao PD, respectivamente; esses resultados também estão associados, provavelmente, às condições físicas do solo, como densidade e porosidade, que interferem nos conteúdos de água e ar do solo e, conseqüentemente, na disponibilidade de nutrientes para as plantas (Vine \& Ahmad, 1987).

Avaliando as condições físicas do solo da área experimental, Cavalieri (2004) observou que essas condições foram mais limitantes no $\mathrm{PD}$, de forma que os sistemas de manejo que propiciem menor densidade, maior porosidade e menor nível de resistência do solo a penetração, tendem a favorecer o desenvolvimento das raízes tuberosas da mandioca; esses fatores devem ter exercido efeito sobre os resultados obtidos neste trabalho, da mesma forma que Oliveira et al. (2001), Souza et al. (1994) e Kang et al. (1998), obtiveram maior produção de raízes tuberosas no PC e PM; em solos leves, com elevado teor de matéria orgânica e com textura média a arenosa, Tonglum et al. (1988) notaram que no PD a produção de raízes tuberosas de mandioca foi similar ou até mais elevada que no PM. Outros estudos desenvolvidos, Cadavid et al. (1998), Hauser et al. (2000) e Aiyelari et al. (2001) não encontraram efeito significativo dos sistemas de preparo do solo sobre a produção de 
raízes tuberosas de mandioca; verifica-se, portanto, que os sistemas de preparo podem apresentar resposta diferenciada em diferentes condições ambientais, o que foi corroborado pela interação significativa entre sistema de preparo e ano, neste trabalho (Tabela 1).

Ao longo dos anos também se constatou redução na produção de raízes tuberosas (Tabela 2); para todos os tratamentos estudados nos dois primeiros anos, o plantio foi efetuado na primeira quinzena de outubro, o que propiciou a extensão do ciclo da cultura, fato este que pode ter contribuído para a obtenção de produtividades de raízes tuberosas mais elevadas. Por sua vez, nos anos 3 e 4 o plantio ocorreu em meados de novembro, com conseqüente redução do ciclo da cultura, fato que resultou na diminuição da produtividade de raízes tuberosas. O efeito das épocas de plantio sobre a produtividade de raízes tuberosas da mandioca foi apresentado por Normanha \& Pereira (1950) e por Takahashi (2002) que indicaram que pode ocorrer redução acentuada da produtividade da cultura da mandioca na região Centro Sul do Brasil, quando a mesma é plantada após o mês de outubro. A redução de produção de raízes tuberosas observada no terceiro e no quarto anos de avaliação, além da época de plantio está associada à ausência de rotação de culturas; salienta-se que, no período de aproximadamente cento e vinte dias entre a colheita da mandioca, o plantio da cultura de inverno e o subseqüente plantio da mandioca, certamente não caracterizaram uma rotação de culturas; portanto, não devem ter propiciado os benefícios inerentes a tal prática ocasionando, como conseqüência, a redução na produção da cultura no terceiro e no quarto ano de avaliação. A redução da produtividade de raízes tuberosas de mandioca, sob monocultivo, também foi reportada pela EMBRAPA (1986).

De forma geral, e embora o PM tenha propiciado uma produtividade inferior ao PC, ele poderá constituir uma opção a ser utilizada pelos agricultores da região Noroeste do Paraná, os quais cultivam mandioca em solos que apresentam elevada susceptibilidade a erosão.

Em relação ao teor de massa seca nas raízes tuberosas da mandioca (Tabela 2), o PC proporcionou teores mais elevados de massa seca nas raízes tuberosas apenas no ano 1 $(\mathrm{P} \leq 0,05)$; nos anos 2 a 4 e na média dos quatro anos de avaliação, os sistemas de preparo não diferiram entre si ( $\mathrm{P}>0,05)$, de forma análoga aos resultados obtidos por Oliveira (1998) e Cadavid et al. (1998).

\section{CONCLUSÕES}

1. O preparo convencional e o preparo mínimo propiciaram maior altura de plantas.

2. O preparo convencional propiciou maior produção de parte aérea e maior produção de raízes tuberosas.

3. O teor de massa seca nas raízes tuberosas não foi influenciado pelo sistema de preparo de solo.

4. O sistema de preparo mínimo pode ser uma opção de sistema de cultivo a ser utilizada a fim de evitar perdas de solo por erosão.

\section{LITERATURA CITADA}

Aiyelari, E. A.; Ndaeyo, U. N.; Agboola, A. A. Effects of tillage practices on growth and yield of cassava (Manihot esculenta) and some soil properties in Ajibode, South-western Nigeria. Indian Journal of Agricultural Sciences, New Delhi, v.71, n.3, p.171-176, 2001.

Cadavid, L. F.; El-Sharkawy, M. A.; Sánchez, A. A. T. Long-term effects of mulch, fertilization and tillage on cassava grown in sandy soils in northern Colombia. Field Crops Research, Amsterdam, v.57, p.45-56, 1998.

Carvalho, F. L. C.; Souza, L. da S.; Caldas, R. C.; Mattos, P. L. P. Efeito da redução do preparo do solo sobre o comportamento produtivo da mandioca. Pesquisa Agropecuária Brasileira, Brasília, v.23, p.609-614, 1988.

Cavalieri, K. M. V. Efeitos de sistemas de preparo nas propriedades físicas de um Latossolo Vermelho distrófico textura média. Maringá: UEM, 2004. 54p. Dissertação Mestrado

Cock, J. H. Aspectos fisiologicos del crecimiento y desarrollo de la planta de yuca. In: Dominguez, C. E. (ed.). Yuca: Investigación, produción y utilización. Cali: CIAT, 1982. p.81-72.

Cruz, C. D.; Regazzi, A. J. Modelos biométricos aplicados ao melhoramento genético. 2.ed. rev. Viçosa: UFV, 2001. 309p.

Domzal, A.; Slowinska-Jurkiewicz, A. Effect of tillage and wheather condition on the structure and physical properties of soil and yield of winter wheat. Soil Tillage Research, Oxford, v.10, p.225-241, 1987.

EMBRAPA - Empresa Brasileira de Pesquisa Agropecuária. Relatório técnico anual do Centro Nacional de Pesquisa em Mandioca e Fruticultura 1985. Cruz das Almas: Embrapa/ CNPMF, 1986.

EMBRAPA - Empresa Brasileira de Pesquisa Agropecuária. Sistema brasileiro de classificação de solos. Brasília: CNPS/SPI, 1999. 412p.

FAO. Food and Agriculture Organization of The United Nations. (On-line). Production. Crops Primary - Cassava. http:// faostat.fao.org/. 12 Jul. 2006

Fidalski, J. Diagnóstico de manejo e conservação do solo e da água na região Noroeste do Paraná. Revista Unimar, Maringá, v.19, p.845-851, 1997.

Godoy, H.; Correia, A. R.; Santos, D. Clima do Paraná. Manual agropecuário para o Paraná. Londrina: IAPAR, 1976. p.17-36.

Grosmann, I.; Freitas, A. C. Determinação do teor de matéria seca pelo peso específico em raízes de mandioca. Revista Agronômica, Porto Alegre, v.14, p.75-80, 1950.

Groxko, M. Secretaria da Agricultura e do Abastecimento. Departamento de Economia Rural. http://www.pr.gov.br/seab. 13 Out. 2006

Hauser, S.; Ndi, J. N.; Hulugalle, N. R. Performance of a maize/ cassava intercrop in tilled and no-till Senna spectabilis alley cropping on an Ultisol in Southern Cameroon. Agroforestry Systems, Dordrecht, v.49, n.2, p.177-188, 2000.

Ismail, M. R.; Davies, W. J. Root restriction affects leaf growth and stomatal response: the role of xylem sap ABA. Scientiae Horticulturae, Amsterdam, v.74, p.257-268, 1998.

Kang, B. T.; Salako, F. K.; Hulugalle, N. R.; Schulte, A.; Ruhiyat, D. Effect of tillage and woody hedgerows in alley cropping on the productivity of an Alfisol. Soils of Tropical Forest Ecosystems: Characteristics, ecology and management, 1998. p.144-149. 
Lorenzi, J. O.; Dias, C. A. C. Cultura da mandioca. Campinas: SAA/CATI, 1993. 41p. Boletim Técnico, 211.

Normanha, E. S.; Pereira, A. S. Aspectos agronômicos na cultura da mandioca (Manihot utilissima, Pohl). Bragantia, Campinas, v.10, n.7, p.179-292, 1950.

Oliveira, J. O. A. P. Efeito de sistemas de preparo em algumas propriedades do solo e na cultura da mandioca (Manihot esculenta, Crantz). Maringá: UEM, 1998. 112p. Dissertação Mestrado

Oliveira, J. O. A. P.; Vidigal Filho, P. S.; Pequeno, M. G.; Scapim, C. A.; Tormena, C. A.; Muniz, A. S.; Sagrilo, E. Influência de sistemas de preparo de solo na produtividade da mandioca ( $M a-$ nihot esculenta, Crantz). Revista Brasileira de Ciência do Solo, Viçosa, v.25, n.2, p.443-450, 2001.

Passioura, J. B.; Gardner, P. A. Control of leaf expansion in wheat seedlings growing in drying soil. Australian Journal of Plant Physiology, Collingwood, v.17, p.149-157, 1990.

Souza, L. da S. Manejo e conservação do solo para a cultura da mandioca. In: Curso intensivo nacional de mandioca, 6, 1986, Cruz das Almas, Anais... Cruz das Almas: EMBRAPA/ CNPMF, 1986. 40p.

Souza, L. da S.; Carvalho, F. L. C. Alterações em propriedades físicas e químicas do solo causadas por sistemas de preparo em mandioca. Revista Brasileira de Mandioca, Cruz das Almas, v.14, p.39-50, 1995.
Souza, L. da S.; Carvalho, F. L. C.; Caldas, R. C.; Mattos, P. L. P. Efeito da profundidade de lavração na cultura da mandioca. I. Componentes do rendimento. Revista Brasileira de Mandioca, Cruz das Almas, v.13, p.137-146, 1994.

Takahashi, M. Implantação da cultura. In: Takahashi, M.; Fonseca Jr., N. S.; Torrecillas, S. M. (Org.). Mandioca no Paraná: antes agora e sempre. Curitiba: IAPAR, 2002. p.59-89. Circular Técnica, 123.

Tonglum, A.; Tiraporn, C.; Sinlhuprama, S. Cassava cultural practice research in Thailand. In: Howeler, R. H.; Kawano, K. (ed.), Cassava breeding and agronomy research in Asia. Rayong: Thayland, 1988. p.131-144.

Vidigal Filho, P. S.; Pequeno, M. G.; Scapim, C. A.; GonçalvesVidigal, M. C.; Maia, R. R.; Sagrilo, E.; Simon, G. A.; Lima, R. S. Avaliação de cultivares de mandioca na região noroeste do Paraná. Bragantia, Campinas, v.59, n.1, p.69-75, 2000.

Vine, P. N.; Ahmad, N. Yield development in cassava under different soil physical conditions. Field Crops Research, Amsterdam, v.17, p.175-198, 1987.

Watanabe, S. H.; Tormena, C. A.; Araújo, M. A.; Vidigal Filho, P. S.; Pintro, J. C.; Costa, A. C. S.; Muniz, A. S. Propriedades físicas de um Latossolo Vermelho distrófico influenciadas por sistemas de preparo do solo utilizados para implantação da cultura da mandioca. Acta Scientiarum - Agronomy, Maringá, v.24, n.5, p.1255-1264, 2002. 